\title{
The Fundus Phenotype Associated with the p.Ala243Val BEST1 mutation.
}

\section{Abbreviated Title: p.Ala243Val BEST1 Fundus Phenotype}

Kamron N Khan PhD, FRCOphth[1-3], Farrah Islam FCPS, FRCS[2], Anthony Robson PhD[4], Graham E Holder PhD[4], Omar AR Mahroo PhD, FRCOphth[1,2], Andrew R Webster MD(Res) FRCOphth[1,2], Anthony T Moore FRCS, FRCOphth[1,2,5], Michel Michaelides MD(Res) FRCOphth[1,2]

1. University College London Institute of Ophthalmology, University College London, London, UK.

2. Inherited Eye Disease Service, Moorfields Eye Hospital, London, UK.

3. Department of Ophthalmology, Leeds Institute of Molecular Medicine, St James's University Hospital, Beckett St, Leeds, UK.

4. Department of Electrophysiology, Moorfields Eye Hospital, London, UK.

5. Ophthalmology Department, University of California San Francisco Medical School, San Francisco, California, USA.

Corresponding authors: Kamron Khan and Michel Michaelides at address 1 above. Email: medknk@leeds.ac.uk and michel.michaelides@ucl.ac.uk Grants/ Financial Disclosure: National Institute for Health Research Biomedical Research Centre at Moorfields Eye Hospital National Health Service Foundation Trust and UCL Institute of Ophthalmology (UK; KNK, ARW, OAM, ATM, MM), Fight For Sight (UK; ARW, MM, OAM), Moorfields Eye Hospital Special Trustees (UK; MM), Moorfields Eye Charity (UK; MM), the Foundation Fighting Blindness (FFB, USA; ARW, ATM, MM), Retinitis Pigmentosa Fighting Blindness (UK; ARW, ATM, MM), and the Wellcome Trust (099173/Z/12/Z; ARW, MM). Michel Michaelides is supported by an FFB Career Development Award. This research has been funded/supported by the National 
Institute for Health Research Rare Diseases Translational Research Collaboration (NIHR RD-TRC). The views expressed are those of the author(s) and not necessarily those of the NHS, the NIHR or the Department of Health."

Proprietary Interest : None

CONFLICT OF INTEREST: No conflicting relationship exists for any of the authors. 


\section{KEY WORDS}

Best Retinopathy.

Best related Retinopathy.

Retina.

Inherited Retinal dystrophy.

\section{SUMMARY STATEMENT}

Best Disease is diagnosed in the presence of a well-defined vitelliform shaped macular lesion. We now describe a second, highly recognisable and reproducible retinal phenotype associated with a specific BEST1 mutation - p.Ala243Val. 


\title{
The retinal phenotype associated with the p.Ala243Val BEST1 mutation.
}

\begin{abstract}
Purpose:

To describe a highly recognisable and reproducible retinal phenotype associated with a specific BEST1 mutation - p.Ala243Val.
\end{abstract}

\section{Methods:}

Retrospective review of consecutive cases where genetic testing has identified p.Ala243Val BEST1 as the cause of disease. Electronic patient records were used to extract demographic, as well as functional and anatomical data. We then compared these data to those observed with the most common BEST1 genotype, p.Arg218Cys.

\section{Results}

Eight individuals (six families) were identified with the p.Ala243Val BEST1 mutation and seven patients with the pathological variant p.Arg218Cys. No patients with mutation of codon 243 knowingly had a family history of retinal disease, whereas all patients with the p.Arg218Cys variant did. The maculopathy was bilateral in all cases. The p.Ala243Val mutation was associated with a pattern dystrophy type appearance, most visible with near infra-red reflectance and fundus autofluorescence imaging. This phenotype was never observed with any other genotype. This mutation was associated with an older median age of symptom onset (median $=42$, IQR $=22$ ) compared with those harbouring the p.Arg218Cys mutation (median = 18, IQR = 12; Mann Whitney U test $\mathrm{p}<0.05$ ). Despite their older age, the final recorded acuity appeared to better in the 
p.Ala243Val group (median $=0.55, \mathrm{IQR}=0.6475 ;$ median $=0.33, \mathrm{IQR}=0.358)$ although this did not reach statistical significance (Mann Whitney U test $p>0.05$ ).

\section{Conclusions}

The mutation p.Ala243Val is associated with a highly recognisable and reproducible pattern dystrophy-like phenotype. Patients develop symptoms at a later age, and tend to have better preservation of EOG amplitudes. 


\section{The Fundus Phenotype Associated With The p.Ala243Val BEST1 mutation.}

\section{Introduction}

Mutations in the BEST1 gene are a common cause of inherited macular disease. ${ }^{1}$ In the majority of cases heterozygous missense variants in the first half of the gene result in a single vitelliform lesion centred on the macula, the classical phenotype associated with Best disease (BD, OMIM 153700).2, 3 Most have a family history consistent with dominant transmission, although non-penetrant carriers and unilateral presentation rarely occur[kk1]. ${ }^{4}$ Less commonly homozygous or compound heterozygous mutations in BEST1 result in recessive disease..$^{5}$ This is usually associated with multifocal subretinal deposit, intraretinal and subretinal fluid accumulation even in the absence of choroidal neovascular membrane, short axial length and narrow irido-corneal angles. ${ }^{6}$ In addition, much rarer phenotypes also have been described (microcornea, rod-cone dystrophy, cataract and posterior staphyloma, autosomal dominant vitreo-retino-choroidopathy (both OMIM 193220) and retinitis pigmentosa (OMIM 613194).7, 8 In addition to causing BD, a maculopathy usually evident in childhood, mutation of BEST1 can also produce a similar appearance later in life, often after the fourth decade. Adult-onset vitelliform macular dystrophy (AVMD, OMIM 608161) is genetically heterogeneous with pathogenic variants in PRPH2, IMPG1 and IMPG2 as well as BEST1 associated with this phenotype. ${ }^{9}$ Perhaps the least well-recognised presentation of BEST1 related retinopathy is a form that mimics the reticular type of pattern dystrophy (PD). ${ }^{10-12}$ Here we present a detailed investigation of a subset of patients presenting with a pattern dystrophy, all found to result from the p.Ala243Val BEST1 mutation. 


\section{Methods}

Patient 4 (see Table 1) presented to the eye clinic at Moorfields Eye Hospital, London and was diagnosed with a macular dystrophy after clinical and electrophysiological examinations. Molecular genetic testing identified p.Ala243Val BEST1 as the causative variant. As the presentation was not typical for $\mathrm{BD}$, we chose to further investigate the phenotype associated with this specific mutation, interrogating an in-house database to identify all patients harbouring this variant. The clinical findings were then compared to those seen with the most prevalent BEST1 mutation, p.Arg218Cys, chosen to serve as an example of "classical" BD. Finally, the phenotype associated with all other BEST1 variants in our database were reviewed for comparison.

Clinical details and patient demographics were reviewed using the patients' hospital notes and electronic patient records (OpenEyes, London, UK). Fundus images included optical coherence tomography (OCT) with accompanying near infrared reflectance imaging (NIR-R) and fundus autofluorescence (FAF), both acquired using the Spectralis HRA and OCT system (Heidelberg Engineering, Heidelberg, Germany).

Electrophysiological testing was performed incorporating the International Society of Clinical Electrophysiology of Vision standards and included full field electroretinogram (ERG), pattern electroretinogram (PERG) and electro-oculogram (EOG). ${ }^{13}, 14$ Molecular testing was performed either by Sanger sequencing (National Genetics Reference Laboratory (NGRL), Manchester, UK) or targeted Next Generation Sequencing of genes known to cause macular dystrophy (Macular panel, Casey Eye Institute, Portland, Oregon, USA). All patients had previously provided informed consent as part of a genetics research project approved by the local research ethics committee, and all investigations were conducted in accordance with the principles of the Declaration of 
Helsinki. The data was analysed using non-parametric statistical methods (Mann Whitney U test) and presented as median plus interquartile range (IQR). Significance was reported testing at the $5 \%$ level.

\section{$\underline{\text { Results }}$}

Eight individuals (six families) were identified with the p.Ala243Val BEST1 mutation and seven patients with the pathological variant p.Arg218Cys (Table 1). No patients with mutation of codon 243 knowingly had a family history of retinal disease, however a family survey in two pedigrees identified one further asymptomatic individual (patients 1a and 2a). Patients carrying the p.Arg218Cys variant could all identify at least one other affected family member. Patients presented with bilateral disease in all cases, independent of their genotype. The p.Ala243Val mutation was associated with an older median age of symptom onset (median $=42, I Q R=22$ ) compared with those harbouring the p.Arg218Cys mutation (median = 18, IQR = 12; Mann Whitney U test $\mathrm{p}<0.05$; see Table 1). Despite their older age, the final recorded acuity appeared to better in the p.Ala243Val group (median $=0.55, \mathrm{IQR}=0.6475 ;$ median $=0.33, \mathrm{IQR}=0.358)$ although this did not reach statistical significance (Mann Whitney U test $\mathrm{p}>0.05$ ).

Fundus examination identified classical changes associated with BD in all patients with mutation of codon 218 - representative of the various stages of disease (Figure 1A). This was confirmed by retinal imaging (SD-OCT and FAF, Figure 1B, C). However, patients with the p.Ala243Val mutation did not always share this typical appearance. A single macular lesion was usually identifiable on fundoscopy, especially early in the disease course (patient 1, Figure 2A). Later, peripheral spokes may have developed, in a pattern dystrophy-like appearance (Figure 2B). 
SD-OCT scans confirmed the presence of subretinal fluid (SRF), a hallmark of BEST1related retinopathy. In addition, small amounts of subretinal deposit were seen to collect preferentially in the periphery of the elevated neurosensory retina, either in a continuous ring of peripheral deposit (Figure 3A), or as radiating spokes (Figure 3B). This material was highly reflective when viewed with NIR-R imaging and most easily visible with this modality (Figure 4A, 4B). In some cases these changes extended into the peripheral macula (Figures 4C). Areas of outer retinal thickening, particularly in the superior retina involving the interdigitating zone were also noted. A similar pattern of change was identifiable on FAF, although less clearly defined (Figure 5A, 5B). Subretinal deposit was associated with hyperautofluorescence, and in some cases a deposit: fluid interface was seen. Again with this modality, the main lesion in most cases did not conform to an approximately circular shape, and often had radial spoke-like extensions. Similar OCT or FAF changes were not observed with the allele p.Arg218Cys.

Electrophysiological testing was available for review in 7/8 cases associated with BEST1 p.Ala243Val. The ffERG was normal in all cases, whilst the EOG and PERG were abnormal in all but one patient who had a normal EOG (Patient 5). The median Arden ratio was 130\% (IQR 24). The absence of a light rise in two cases (Patients 3 and 4) directed genetic screening towards BEST1. In contrast the p.Arg218Cys mutation was always associated with a reduced Arden ratio in the $4 / 7$ cases tested, with a median amplitude of $117.5 \%$ (IQR 10). These data suggest that this specific mutation of codon 243 may have less impact on the amplitude of the EOG light rise than the p.Arg218Cys variant (Mann Whitney $U$ test $\mathrm{p}<0.05$ ).

In an attempt to discover whether this specific phenotype is seen in the context of other BEST1 genotypes we then reviewed the retinal images of other patients, identified from 
an in-house database, known to have molecularly proven BD. The retinal appearance resulting from p.Ala243Val BEST1 was not reproduced by more than 20 further genotypes.

\section{Discussion}

In this work we describe a highly recognisable and reproducible retinal phenotype associated with a specific BEST1 mutation - p.Ala243Val. The unique anatomical changes are best identified with NIR-R imaging, in addition to SD-OCT and FAF. This variant appears milder than others causes of $\mathrm{BD}$, as patients develop symptoms at a later age, and tend to have better preservation of EOG amplitudes. These data will be invaluable both clinically, aiding the diagnosis of new cases, and scientifically, when attempting to better understand this protein's function.

So far over 200 mutations in BEST1 have been described, most of which are heterozygous and associated with a vitelliform macular dystrophy (http://wwwhuge.uni-regensburg.de/BEST1_database). BD in its classical form is a childhood-onset

condition characterised by the accumulation of subretinal deposit centred on the fovea. ${ }^{4}$ Causative genetic variants almost exclusively occur in the highly conserved amino terminal domain of BEST1 (amino-acids 1-390) and it is thought that missense mutations causing BD act by exerting a dominant negative effect, altering calcium sensitive chloride conductance through this channel. ${ }^{15}$ BEST1 haploinsufficiency, as occurs in carriers of recessive BEST1 disease, is not associated with any detectable retinopathy. The phenotype arising from the p.Ala243Val mutation is milder than classical BD, with a later age of visual loss and absence of a large volume of central deposit. 
The substitution p.Ala243Val has been previously identified as a cause of BD, AVMD and in one report PD. Kramer et al describe three pedigrees with BD and five with AVMD arising from mutation of codon $243 .{ }^{16}$ Unusually the patients with BD were older than expected (most were symptomatic after the age of 30) and the sole paediatric patient aged 15 showed only the earliest signs of disease (described by the authors as pre-vitelliform stage). Although clinical images are not available, the description is that of a milder disease than is usual for BD. BD has also been reported once before with the same genotype, by Chung et al. in $2001 .{ }^{17}$ This patient was seen aged 23 with a unilateral gliotic macular scar presumed to result from prior choroidal neovascularisation. There was no comment on the fellow eye. The same mutation was reported by Querques et al. in 2009 where they describe five members of a single family. ${ }^{18}$ No fundus images are available for review. The youngest mutation carriers (aged 13 and 17) had not yet developed a visible phenotype, again perhaps suggesting a disorder with a later onset. Lastly, and in the only case where fundus images were published, Boon et al. describe a patient with a pattern dystrophy-type maculopathy due to the p.Ala243Val mutation. ${ }^{19}$ In this case, and most of the cases herein, the Arden ratio was found to be larger than that usually associated with BD. The clinical evidence presented therefore supports a role for this variant having a milder effect than most heterozygous mutations in BEST1.

The BEST1 gene encodes a channel protein that selectively transports anions and localises to the basolateral RPE cell membrane. ${ }^{20-23}$ Recently however an alternative location has been suggested. Singh et al. generated mutant RPE cells using induced pluripotent stem cells derived from fibroblasts of patients with BD. ${ }^{24}$ They propose that the channel is sited in the RPE cytosol, near to but not actually in the basolateral 
membrane, probably residing in the endoplasmic reticulum (ER). Whilst its preference is for chloride ions the channel may additionally transport other monovalent anions (bromine, iodine, thyocynate, bicarbonate and nitrate) and even glutamate and GABA. ${ }^{25}$ In the ER it is thought to have a role in calcium homeostasis. Yu et al. investigated the functional consequences of the p.Ala243Val variant in patch clamping studies, overexpressing the mutated BEST1 protein in HEK293 cells. ${ }^{26}$ The currents detected were approximately $10 \%$ as large as those measured through wild-type channels. ${ }^{26}$ They, and others, were also able to show that p.Ala243Val BEST1 is expressed on the cell surface at approximately the same level as wild-type BEST1, suggesting that this missense mutation reduces ion conductance through the channel rather than mislocalising the channel. ${ }^{26} 27,28$ These data are also in keeping with a hypothesis where the effects of mutation are to alter normal function. A more dramatic change in function, perhaps even a reversal of polarity, may be associated with mutation of key pore forming residues. ${ }^{29}$

Whilst it is easy to understand what consititutes a milder form of disease, one with a later onset and less pronounced maculopathy, it is not intuitive how this is brought about at a molecular level. A major difference between the PD and vitelliform phenotypes is the reduced quantity of subretinal deposit in the former, as well as an altered distribution. The accumulating material in classical BD is usually highly autofluorescent, and derives from undigested photoreceptor outer segments loaded with bisretinoid product that are thought to accumulate due to impaired phagocytosis. A similar phenotype occurs with mutation of $P R P H 2$, where mutated photoreceptor outer segments are either shed in greater numbers or are more resistant to RPE phagocytosis. ${ }^{9}$ Lastly, mutation of IMPG1 and IMPG2 can also generate a vitelliform 
maculopathy, and again it is suggested that impaired clearance of outer segments is the cause. ${ }^{30}$ Here the p.Ala243Val mutation is associated with significant amounts of subretinal fluid, even in the earliest stages of disease. It may well be that detachment of the neurosensory retina is the primary event, and photoreceptor outer segments accumulate as a consequence, in a manner not dissimilar to that observed with another retinal disease - central serous chorioretinopathy. This mutation may therefore impair a specific aspect of RPE function, uniquely altering ion flux and the associated osmotic movement of water, whilst a different physiological process may be relatively spared phagocytosis of photoreceptor outer segments. Although the genetic mechanisms responsible are widespread, present in all RPE cells, the clinical phenotype is likely to be influenced by both variations in regional metabolic activity (macular versus peripheral retina) and the differences between popultaions of RPE cells even within the macula. As RPE cells are understood to migrate centrifugally during ocular organogenesis, radial patterns of central RPE disease have long been identified (eg. dominant drusen in association with mutation of EFEMP1). Small amounts of subretinal deposit may therefore accumulate in a pattern that is determined primarily by clonal origin, with little influence of gravity. Most cases of BD however are associated with larger subretinal collections; under these circumstances a predisposition to radially distributed deposit is lost, and the greater volume of subretinal material is primarily influenced by gravity.

In summary, this study describes a new retinal phenotype associated with the p.Ala243Val BEST1 genotype. Our study demonstrates the association of a reticular pattern dystrophy-like appearance with mutation in BEST1, in addition to the long recognised association with $P R P H 2$, and so should be considered in the differential 
diagnosis. This specific mutation is also associated with a later age of disease onset, and with greater EOG amplitudes than are usually associated with BD. These clinical characteristics may provide mechanistic insight, suggesting that the p.Ala243Val mutation acts primarily to impair ion transport and the associated movement of fluid, with minimal disruption to RPE phagocytosis, resulting in a later-onset maculopathy. Family surveys may be necessary in order to identify similarly affected individuals. 


\section{REFERENCES}

1. Boon CJF et al. The spectrum of ocular phenotypes caused by mutations in the BEST1 gene. Progress in retinal and eye research 2009; 28:187-205.

2. Petrukhin $\mathrm{K}$ et al. Identification of the gene responsible for Best macular dystrophy. Nature genetics 1998; 19:241-247.

3. Marquardt A et al. Mutations in a Novel Gene, VMD2; Encoding a Protein of Unknown Properties Cause Juvenile-Onset Vitelliform Macular Dystrophy (Best's Disease). Human molecular genetics 1998; 7:1517-1525.

4. Boon CJ et al. The spectrum of ocular phenotypes caused by mutations in the BEST1 gene. Progress in retinal and eye research 2009; 28:187-205.

5. Burgess $R$ et al. Biallelic mutation of BEST1 causes a distinct retinopathy in humans. The American Journal of Human Genetics 2008; 82:19-31.

6. Boon CJ et al. Autosomal recessive bestrophinopathy: differential diagnosis and treatment options. Ophthalmology 2013; 120:809-820.

7. Yardley $\mathrm{J}$ et al. Mutations of VMD2 splicing regulators cause nanophthalmos and autosomal dominant vitreoretinochoroidopathy (ADVIRC). Investigative ophthalmology \& visual science 2004; 45:3683-3689.

8. Davidson AE et al. Missense mutations in a retinal pigment epithelium protein, bestrophin-1, cause retinitis pigmentosa. The American Journal of Human Genetics 2009; 85:581-592.

9. Chowers I et al. Adult-onset foveomacular vitelliform dystrophy: a fresh perspective.

Progress in retinal and eye research 2015; 47:64-85.

10. Kramer $\mathrm{F}$ et al. Mutations in the VMD2 gene are associated with juvenile-onset vitelliform macular dystrophy (Best disease) and adult vitelliform macular dystrophy but not age-related macular degeneration. European Journal of Human Genetics 2000; 8:286-292.

11. White K, Marquardt A and Weber BH. VMD2 mutations in vitelliform macular dystrophy (Best disease) and other maculopathies. Human mutation 2000; 15:301.

12. Seddon JM et al. Assessment of mutations in the Best macular dystrophy (VMD2) gene in patients with adult-onset foveomacular vitelliform dystrophy, age-related maculopathy, and bull'seye maculopathy. Ophthalmology 2001; 108:2060-2067.

13. Bach M et al. ISCEV standard for clinical pattern electroretinography (PERG): 2012 update. Documenta Ophthalmologica 2013; 126:1-7.

14. McCulloch DL et al. ISCEV Standard for full-field clinical electroretinography (2015 update). Documenta ophthalmologica 2015; 130:1-12.

15. Strauß O et al. The Role of Bestrophin-1 in Intracellular Ca2+ Signaling. Retinal Degenerative Diseases. Springer, 2014; 113-119.

16. Krämer $\mathrm{F}$ et al. Ten novel mutations in VMD2 associated with Best macular dystrophy (BMD). Human mutation 2003; 22:418-418.

17. Chung MM et al. Visual outcome following subretinal hemorrhage in Best disease. Retina (Philadelphia, Pa) 2001; 21:575-580.

18. Querques $\mathrm{G}$ et al. Functional and clinical data of Best vitelliform macular dystrophy patients with mutations in the BEST1 gene. 2009. 
19. Boon $\mathrm{CJ}$ et al. Clinical and molecular genetic analysis of best vitelliform macular dystrophy. Retina (Philadelphia, Pa) 2009; 29:835-847.

20. Marmorstein AD et al. Bestrophin, the product of the Best vitelliform macular dystrophy gene (VMD2), localizes to the basolateral plasma membrane of the retinal pigment epithelium. Proceedings of the National Academy of Sciences 2000; 97:12758-12763.

21. Gouras $P$ et al. Bestrophin detected in the basal membrane of the retinal epithelium and drusen of monkeys with drusenoid maculopathy. Graefe's Archive for Clinical and Experimental Ophthalmology 2009; 247:1051-1056.

22. Hu J and Bok D. A cell culture medium that supports the differentiation of human retinal pigment epithelium into functionally polarized monolayers. Mol Vis 2001; 7:14-19.

23. Ablonczy $Z$ et al. Human retinal pigment epithelium cells as functional models for the RPE in vivo. Investigative ophthalmology \& visual science 2011; 52:8614-8620.

24. Singh $R$ et al. iPS cell modeling of Best disease: insights into the pathophysiology of an inherited macular degeneration. Human molecular genetics 2012:dds469.

25. Dickson VK, Pedi L and Long SB. Structure and insights into the function of a Ca2+-activated Cl-channel. Nature 2014; 516:213-218.

26. Yu K, Cui Y and Hartzell HC. The bestrophin mutation A243V, linked to adult-onset vitelliform macular dystrophy, impairs its chloride channel function. Investigative ophthalmology \& visual science 2006; 47:4956-4961.

27. Milenkovic VM et al. Insertion and topology of normal and mutant bestrophin-1 in the endoplasmic reticulum membrane. Journal of biological chemistry 2007; 282:1313-1321.

28. Johnson AA et al. Disease-causing mutations associated with four bestrophinopathies exhibit disparate effects on the localization, but not the oligomerization, of Bestrophin-1. Experimental eye research 2014; 121:74-85.

29. Yang T et al. Structure and selectivity in bestrophin ion channels. Science 2014; 346:355-359. 30. Meunier I et al. Frequency and clinical pattern of vitelliform macular dystrophy caused by mutations of interphotoreceptor matrix IMPG1 and IMPG2 genes. Ophthalmology 2014; 121:24062414. 


\section{LEGENDS}

Figure 1. Patient 4 (p.Arg218Cys) displaying the classical changes of advanced Best Disease. Fundus photography reveals central macular atrophy in both eyes (A), which is clearly defined by fundus autofluorescence imaging (FAF), with the additional finding of hyperautofluorescent deposit at the most inferior aspect (B). These changes are easily identifiable by optical coherence tomography (OCT).

Figure 2. Retinal appearance with mutation of p.Ala243Val BEST1 (colour fundus photography). Right and left eyes of Patient 1, aged 18, showing a single lesion early in the disease course (A). Right and left eyes of Patient 2, aged 55. Peripheral spoke-like components of the lesion are now visible, the most common finding in this age group of patients (B).

Figure 3. Near infrared reflectance imaging (NIR-R) and corresponding optical coherence tomography scans for patient 3 . The right eye has a predominantly circumferential distribution of deposit (A) whilst the left eye has additional peripheral spokes (B).

Figure 4. Near infrared reflectance imaging (NIR-R) in right and left eyes of patient 6 (A), patient 4 (B), and patient 2 (C) demonstrating the variation in phenotype associated with different patterns of subretinal deposit.

Figure 5. Fundus autofluorescence (FAF) (A) and near infrared reflectance imaging (NIR-R) (B) for patient 4 (left hand images) and patient 6 (right hand images). Each modality identifies a slightly different feature of disease - NIR-R appears to be particularly sensitive to subtle separation of the neurosensory retinal from the RPE, and particularly a change in orientation of the ellipsoid zone, with increased reflectivity evident in regions with no or minimal change in autofluorescence (white arrows). FAF appears to be a more sensitive technique for detecting larger collections subretinal deposit (red arrows). 